MONS. ANTONIO INTERGUGLIELMI

Studium Theologicum Galilaeae, Israel

\title{
IL VESCOVO COME TESTIMONE DELLA CARITÀ. PROFILI CANONICI
}

Sommario: Premessa: i beni nel diritto canonico nella prospettiva della Carità. - 1. Il Magistero Pontificio. - 2. La gestione dei beni e delle opere della diocesi: cann. 331-333 e 1273. - 3. La funzione di vigilanza del vescovo diocesano nella prospettiva della carità (can. 1276). - 4. La funzione di incentivo alla carità attraverso gli organismi diocesani. - Conclusioni.

\section{Premessa: i beni nel diritto canonico nella prospettiva della Carità}

Nel Codice di diritto canonico al canone 1254 si afferma il diritto nativo della Chiesa a possedere e orientare i beni temporali ai fini che le sono propri: culto, sostentamento dei ministri, apostolato e servizio dei poveri. Il conseguimento di queste finalità giustifica il possesso e l'uso di beni economici mobili e immobili.

La Chiesa, operando nell'umano, ha dunque una vita economica: il Codice di diritto canonico che tratta dei beni temporali della Chiesa (cf. Libro V, cann. 1254-1310), dà alcuni orientamenti sull'uso evangelico dei beni ${ }^{1}$. In esso è detto (cfr. can. 222) che le ragioni e gli orientamenti della vita economica nella Chiesa sono l'attuazione delle realtà intrinsecamente spirituali ossia il culto, l'onesto sostentamento

${ }^{1}$ A. Del Portillo, Laici e fedeli nella Chiesa, Milano 1999, p. 227, sottolinea il fatto che la Chiesa deve possedere beni come «necessità della sua propria natura, giacché si trova inserita nel mondo». 
dei ministri, le opere di apostolato e di carità, anzitutto a servizio dei poveri.

Sono le finalità pastorali che devono ispirare le scelte economiche e non viceversa. Per quanto riguarda i canoni del Codice di diritto canonico che si occupano dei beni, ad uno sguardo superficiale, potrebbe sembrare che esso disciplini la povertà sotto l'aspetto individuale e comunitario ${ }^{2}$, ma che non preveda un obbligo giuridicamente sanzionato di sovvenire alle necessità dei poveri.

Negli ultimi anni la questione riguardante la gestione dei beni della Chiesa ad uso caritativo si è accompagnata ad una revisione, in chiave di chiarezza e trasparenza, della questione economica ecclesiale, in primis a livello centrale, a cui ha dato inizio il Chirografo di Papa Francesco del 18 luglio 2013, con cui il Sommo Pontefice ha previsto l'istituzione di una Pontificia Commissione referente di studio e di indirizzo sull'organizzazione della struttura economicoamministrativa della Santa Sede ${ }^{3}$.

2 Troviamo le norme più complete infatti per quanto riguarda la povertà di coloro che professano i Consigli Evangelici: si tratta delle norme relative alla disciplina dei religiosi, degli istituti di vita consacrata e delle società di vita apostolica.

Anche se le modalità con cui si debba concretamente osservare il consiglio evangelico di povertà è rimesso alle costituzioni dei singoli istituti (cfr. can. 598), il codice stesso al canone 600 ne dà una definizione: "Il consiglio evangelico della povertà, a imitazione di Cristo che essendo ricco si è fatto povero per noi, oltre a una vita povera di fatto e di spirito da condursi in operosa sobrietà che non indulga alle ricchezze terrene, comporta la limitazione e la dipendenza nell'usare e nel disporre dei beni, secondo il diritto proprio dei singoli istituti»

${ }^{3} \mathrm{Nel}$ chirografo di Papa Francesco si leggeva: «Noi abbiamo deciso di istituire una Commissione referente che raccolga puntuali informazioni sulle questioni economiche interessanti le Amministrazioni vaticane e cooperi con il sopra citato Consiglio dei Cardinali nel suo apprezzato lavoro, offrendo il supporto tecnico della consulenza specialistica ed elaborando soluzioni strategiche di miglioramento, atte ad evitare dispendi di risorse economiche, a favorire la trasparenza nei processi di acquisizione di beni e servizi, a perfezionare l'amministrazione del patrimonio mobiliare e immobiliare, ad operare con sempre maggiore prudenza in ambito finanziario, ad assicurare una corretta applicazione dei principi contabili ed a garantire assistenza sanitaria e previdenza sociale a tutti gli aventi diritto". Comunicato della Segreteria di Stato, da www.vatican.va. Si può approfondire in M. A. Cozzolino, Primi 
Il Cardinal Pell, Cardinale Prefetto della Segreteria per l'Economia, ha scritto che nella Chiesa "L'attività amministrativa non va considerata, come è attualmente, relegata in un ghetto e appannaggio di pochi, ma si caratterizza quale privilegiato servizio ecclesiale tout court di natura pastorale su cui si gioca gran parte della credibilità della Chiesa, della sua capacità di testimonianza e di attrazione di nuove risorse necessarie alla sua missione"4.

Nel Codice di diritto canonico, al già ricordato canone 1254, viene precisato che "il "modo" di raggiungere le finalità proprie della Chiesa attraverso i beni dovrà chiaramente mostrare di attuare il criterio discriminante tra povertà e accumulo; allo stesso modo, inoltre, si dovrà perseguire la diffusione, nella vita ecclesiale, di una mentalità che ricerchi la cosiddetta "perequazione comunionale"5.

Le strutture di partecipazione alla vita economica ecclesiale previste nel CIC del 1983, in attuazione del Concilio Vaticano II, i Consigli per gli Affari economici, sia quello diocesano sia quelli parrocchiali, sono infatti prima di tutto organi con una natura pastorale, in quanto la loro funzione si radica in una mentalità ecclesiale sottostante.

E' dunque al Vescovo diocesano, che governa la Chiesa particolare che gli è stata affidata con la potestà piena, legislativa, amministrativa e giudiziaria (can. $391 \$ 1$ ), nonché attraverso l'esercizio del suo ufficio pastorale, su cui incombe questa missione caritativa, orientando l'azione dei presbiteri, dei fedeli e delle associazioni della sua Chiesa (can. $318 \$ 1$ ).

Sarà il Vescovo a garantire un pieno esercizio della carità, ad essere cioè nella Chiesa particolare a lui affidata la guida anche nella carità

interventi di Papa Francesco per una riforma degli organismi economico-finanziari della Curia romana: spunti di riflessioni, in: AA.VV., Recte sapere. Studi in onore di Dalla Torre, a cura di Geraldina Boni, Erminia Camassa, Paolo Cavana, Pasquale Lillo, Vincenzo Turchi, Torino 2014, p. 229-242.

${ }^{4}$ G. Pell, in: AA.VV., Corresponsabilità e trasparenza nella gestione dei beni della Chiesa, a cura di Francesco Lozupone, Roma 2015, Prefazione, pp. 11-14.

${ }^{5}$ Cfr. Il documento del Pontificio Consiglio della Giustizia e della Pace, Aspetti sociali ed etici dell'Economia, un colloquio in Vaticano, Città del Vaticano 1994. 
verso i più bisognosi ${ }^{6}$. Questo lo farà in due ambiti: attraverso la sua funzione di governo, che si esprime con la triplice potestà, nonché mediante la predicazione, l'incoraggiamento e il sostegno di tutte quelle iniziative che si esprimono nell'esercizio della carità, ai più poveri e ai più bisognosi.

Di questo compito ne troviamo accenni nel Codice di diritto canonico in un altro canone:

"Il Vescovo diocesano, consapevole di essere tenuto ad offrire un esempio di santità nella carità, nell'umiltà e nella semplicità di vita, si impegni a promuovere con ogni mezzo la santità dei fedeli, secondo la vocazione propria di ciascuno, ed essendo il principale dispensatore dei misteri di Dio, si adoperi di continuo perché i fedeli affidati alle sue cure crescano in grazia mediante la celebrazione dei sacramenti e perché conoscano e vivano il mistero pasquale" (can. 387).

Anche la Chiesa locale è dunque tenuta a questa primaria funzione della carità nella gestione dei beni che sono affidati al Vescovo diocesano: ma si tratta di un obbligo pastorale previsto anche come vero obbligo giuridico? Se infatti non c'è dubbio che la Chiesa locale nel suo insieme e nei diversi soggetti in cui si articola (parrocchie e ogni altro ente con personalità canonica e civile o anche solo canonica, non esclusi movimenti, associazioni ecc.), dovrà farsi carico di questa primaria funzione pastorale dei beni (perché le risorse economiche sono dentro la pastorale medesima e non un aspetto separato), si può parlare di un obbligo giuridico?

\section{Il Magistero Pontificio}

Sono innumerevoli i richiami e le esortazioni di Papa Francesco che esortano ad un uso dei beni della Chiesa per la carità: «Una Chiesa

${ }^{6}$ «La testimonianza evangelica esige che le opere siano gestite in piena trasparenza, nel rispetto delle leggi canoniche e civili, e poste a servizio delle tante forme di povertà». Così si legge nel documento della CoNGREgAzIONE PER GLI IsTITUTI DI Vita CONSACRATA E Le Società di vita Apostolica, la Lettera Circolare "Linee orientative per la gestione dei beni negli Istituti di vita consacrata e nelle Società di vita apostolica", Città del Vaticano 2014, p. 10. 
senza la carità non esiste - disse il Papa accogliendo in udienza il Comitato esecutivo di Caritas Internationalis ${ }^{7}$ - la Caritas è la carezza della Chiesa al suo popolo; la carezza della Madre Chiesa ai suoi figli; la tenerezza, la vicinanza».

Anche Papa Benedetto XVI aveva messo in luce questo compito caritativo fondamentale della Chiesa, quasi come una preparazione allo sviluppo dell'attuale Pontificato di Papa Francesco, ricordando in particolare che si tratta di uno dei compiti primari per i Vescovi. Nella Lettera enciclica Deus caritas est leggiamo infatti: «alla struttura episcopale della Chiesa [...] corrisponde il fatto che, nelle Chiese particolari, $i$ Vescovi quali successori degli Apostoli portino la prima responsabilità della realizzazione» del servizio della carità (n. 32) e continua sottolineando però che «il Codice di Diritto Canonico, nei canoni riguardanti il ministero episcopale, non tratta espressamente della carità come di uno specifico ambito dell'attività episcopale» (ibidem).

Già nel Direttorio per il ministero pastorale dei Vescovi ${ }^{10}$ del 2004, veniva approfondito e concretizzato il dovere della carità, quale compito intrinseco del Vescovo nella sua Diocesi:

\footnotetext{
${ }^{7}$ Un'espressione dell'attenzione della Chiesa per i più poveri e bisognosi va considerato il riconoscimento di Caritas Internationalis del 19 settembre 2004 da parte di Papa Giovanni Paolo II che, con il Pontificium Consilium Cor Unum, sono gli organismi a livello centrale della Chiesa per l'esercizio della carità. Scrive su questo aspetto G.P. DAL Toso, La relazione di Caritas Internationalis con il Pontificio Consiglio Cor Unum a seguito del Chirografo "Durante l'Ultima Cena, in: AA.VV., Diritto Canonico e servizio della carità, a cura di J. Miñambres, Roma, 2008, p. 405: "Nel chirografo il Sommo Pontefice concede la personalità giuridica canonica pubblica a Caritas Internationalis. Il Can. $116 \$ 1$ precisa che le persone pubbliche sono quelle che realizzano i loro scopi «In nomine Ecclesiae»... essa comporta il riconoscimento di una responsabilità ecclesiale, insieme ad un contrappeso istituzionale".

${ }^{8}$ PAPA Francesco, Udienza ai rappresentanti di Caritas Internationalis, 16 maggio 2013.

${ }^{9}$ Benedetto XVI, Lettera Enciclica Deus caritas est, 25 dicembre 2005, AAS 98 (2006).

${ }^{10}$ Congregazione Per I vescovi, Direttorio per il ministero pastorale dei vescovi, LEV, Città del Vaticano 2004.
} 
«La responsabilità del Vescovo nell'ambito della carità appare fin dalla liturgia dell'ordinazione episcopale, quando al candidato viene posta la domanda specifica: "Vuoi essere sempre accogliente e misericordioso, nel nome del Signore, verso i poveri e tutti i bisognosi di conforto e di aiuto?»" .

E' dunque necessario analizzare anche dal punto di vista canonico come il Vescovo è tenuto ad orientare il servizio della carità nella sua Chiesa, costitutivo del ministero episcopale, tratteggiando i profili giuridici del suo compito di amministratore della Diocesi e come possa quindi attuarla, in primo luogo attraverso le strutture che il Codice prevede a sostegno della funzione amministrativa e di governo della diocesi. In qualche modo il Vescovo dovrà farsi garante perché $i$ beni della Chiesa a lui affidata vengano usati come manifestazione del servizio della Chiesa ai più poveri, espressione della Carità di Cristo Signore.

Nello svolgimento del compito di amministratore della diocesi, il Vescovo dovrà infatti avere un'attenzione particolare per tutte quelle situazioni di bisogno della porzione del popolo di Dio a lui affidata, esercitando questo compito attraverso il suo ministero di amministratore della chiesa particolare - che si esprime innanzitutto nella presidenza degli organismi diocesani, come il Consiglio affari economici (diocesano) - incentivando le istituzioni ecclesiali (Fondazioni e Caritas) e le organizzazioni ecclesiali che si occupano della carità (cfr. can 1277) $)^{12}$.

Vediamo in particolare i diversi ambiti.

\footnotetext{
${ }^{11}$ Op. ult. cit., n. 194.

12 «El patrimonio como conjunto de bienes que, como tales, tienen una utilidad económica no puede concebirse al margen de la condición jurídica de aquellos, pues estos bienes están ordenados, como término objetivo de relaciones jurídicas, a la satisfacción de determinados intereses potegidos por el Derecho», così $\mathrm{M}$. Lopez Alarcon, Apuntes para una teoria general del patrimonio eclesiastico, Ius Canonicum (1962), p. 120.
} 


\section{La gestione dei beni e delle opere della Diocesi: cann. 331-333 e 1273}

Nel Codice di diritto canonico si affermano i seguenti principi:

1. l'unitarietà del patrimonio ecclesiastico pur nella attribuzione alle diverse persone giuridiche di singoli beni (cann. 1255-1258). Queste persone giuridiche sono enti eretti che agiscono in nome e per le finalità proprie della Chiesa (diocesi, parrocchie, istituti di vita consacrata, seminari, confraternite, fondazioni...), ma con il "coordinamento" del Vescovo diocesano (cfr. can 1279). Il Codice afferma altresì che il Romano Pontefice è il "supremo amministratore e dispensatore di tutti i beni ecclesiastici” (can. 1273), poiché il governo dei beni è gerarchicamente ordinato per il fatto che "con il nome di Chiesa si intende non soltanto la Chiesa universale o la Sede Apostolica, ma anche qualsiasi persona giuridica pubblica nella Chiesa" (can. 1258).

2. allo stesso tempo nel Codice viene riconosciuta una certa $a \boldsymbol{u}$ tonomia statutaria delle singole persone giuridiche secondo le disposizioni del Libro $\mathrm{V}$ del Codice, il quale fa espresso riferimento ai "propri statuti" (can. $1257 \$ 2$ ).

In questo quadro il Vescovo ha due fondamentali compiti:

a) E' l'amministratore dell'ente diocesi;

b) deve emettere norme in tema di gestione economica dei beni ecclesiali, al fine di indirizzare e vigilare sull'amministrazione dei beni temporali delle persone giuridiche che dipendono dalla diocesi (can. $1276 \$ 1$ e 2).

Queste funzioni sono regolate dal Libro V del Codice, in particolare dai cann. 492-494 sul Consiglio per gli affari economici e sull'economo diocesano, nonché dalle norme regolamentari delle diverse Conferenze Episcopali nazionali.

Abbiamo già sottolineato che nell'amministrazione del patrimonio ecclesiastico si pone l'accento sul valore essenzialmente strumentale che i beni temporali hanno nella vita della Chiesa, in quanto l'amministrazione è, definiti come l'insieme delle attività che hanno come scopo l'organizzazione del patrimonio riguardo a un fine. 
La Chiesa possiede e amministra i beni temporali solo perché mezzi per raggiungere gli obiettivi che le sono propri: la gestione del culto divino, l'onesto sostentamento del clero e degli altri ministri, il sostegno delle opere di apostolato e di carità (cf. Presbyterorum Ordinis n. 17 e can. $1254 \$ 2)^{13}$.

Il raggiungimento di queste finalità suscita e giustifica una particolare attenzione da parte del diritto della Chiesa per la corretta amministrazione dei suoi beni. Così la responsabilità del patrimonio ecclesiastico è compito specifico del Vescovo insieme ai suoi collaboratori, in primis l'economo diocesano e il Consiglio diocesano affari economici (cann. 494 e 1278).

Considerata la sempre maggiore complessità del mondo economicoamministrativo, si esige la collaborazione di persone qualificate ed esperte che, con il loro prezioso aiuto e sapiente consiglio, favoriscano una buona e corretta amministrazione dei beni e una più razionale e moderna gestione degli stessi ${ }^{14}$.

Da quanto detto, sarà dunque il Vescovo a poter e dover orientare la gestione e l'uso dei beni per finalità caritative: in primo luogo lo farà con quelli che dipendono direttamente da lui.

In ogni diocesi una serie di beni e fondazioni sono gestite direttamente dal Vescovo attraverso la sua Curia: nell'attribuzione delle rendite sarà dunque sua cura principale il finanziamento delle opere di carità, spesso gestite attraverso la Caritas diocesana ma

\footnotetext{
${ }^{13}$ Qui si aprirebbe il problema della legittimità canonica della tassa per la Chiesa prevista in alcune nazioni, come la Germania e l'Austria. Sul punto cfr. E. Corecco, Dimettersi dalla Chiesa per ragioni fiscali, Apollinaris (1982), pp. 461-502, dove scrive: "Bisogna da ciò concludere che nella nuova normativa canonica, un atto formale di dimissioni dalla Chiesa non è previsto né operante giuridicamente se non coinvolge nello stesso tempo il livello della fede. E dunque evidente che un atto di dichiarazione di dimissioni dalla Chiesa per ragioni fiscali non può essere messo «tout-court» in relazione con il fatto dell'appartenenza alla Chiesa. Dev'essere innanzitutto valutato come manifestazione della volontà di non voler assolvere certi doveri finanziari e, di conseguenza, sembra che non sia possibile punirlo con le stesse misure applicabili all'apostasia, all'eresia e allo scisma".

${ }^{14}$ J.P. Schouppe, Elementi di diritto patrimoniale canonico, Giuffrè 1997, p. 151 ss.
} 
anche attraverso altre organizzazioni preposte all'esercizio della beneficenza e assistenza verso i più bisognosi. In alcune diocesi, ad esempio, sono stati costituiti dei Fondi di solidarietà, per finanziare specifiche esigenze di carità: migranti, famiglie senza lavoro, sostegno a situazioni di indebitamento, etc.

$E^{\prime}$ in questa gestione diretta che si esprime più compiutamente l'esercizio esemplare della carità del Vescovo: l'utilizzo delle risorse della diocesi per le opere caritative è infatti la migliore e più efficace testimonianza che i soldi per la Chiesa sono solo "uno strumento" per svolgere la missione di sostegno delle persone più povere.

\section{La funzione di vigilanza del Vescovo diocesano nella prospettiva della carità (can. 1276)}

Il Vescovo è tenuto alla vigilanza sull'amministrazione di tutti $i$ beni ecclesiastici ${ }^{15}$ della sua diocesi:

Can. 1276 «\$ 1. Spetta all Ordinario di vigilare con cura sulla amministrazione di tutti $i$ beni appartenenti alle persone giuridiche pubbliche a lui soggette, salvo titoli legittimi per $i$ quali gli si riconoscano più ampi diritti. \$2. Gli Ordinari, tenuto conto dei diritti, delle legittime consuetudini e delle circostanze, abbiano cura di ordinare l'intero complesso dell 'amministrazione dei beni, dando speciali istruzioni entro i limiti del diritto universale e particolare».

Nello spirito del Codice il dovere della vigilanza del Vescovo diocesano, in maniera primaria rivolta alle Parrocchie, si avvale di alcuni strumenti:

\footnotetext{
${ }^{15}$ Ricordiamo che il Codice definisce Bene Ecclesiastico quello appartenente ad un’associazione pubblica di fedeli (can. $301 \$ 3$ ). Sulle altre associazioni di fedeli che godono di personalità giuridica vige la normativa di cui ai cann. 112-123 e al can. 1255: ogni persona giuridica (pubblica o privata) è un soggetto capace di acquistare, possedere, amministrare ed alienare beni ecclesiastici, in conformità del diritto. Su tutte le associazioni private è comunque competenza del Vescovo una vigilanza, perché i beni siano utilizzati in conformità degli Statuti e della finalità. Cfr. V. DE PAOLIs, Schema canonum libri V. De iure patrimoniali Ecclesiae, Periodica 68 (1979), pp. 673-713.
} 
1. la visita pastorale è un'occasione privilegiata per attivare gli organismi di partecipazione in tutte le parrocchie e permette di aprire un coordinamento nella gestione dei beni e della destinazione delle risorse, stabilendo un'unità tra il progetto pastorale delle singole comunità e quello della diocesi;

2. l'avvicendamento degli amministratori (innanzitutto i parroci) che comporta consegne e inventari a norma del canone 1283, $2^{\circ}$ e $3^{\circ}$ : questo aspetto è della massima utilità perché il Vescovo deve avere una conoscenza quanto più precisa dei beni ecclesiali della sua diocesi, nonché delle risorse che potrà così decidere di destinare a fini caritativi;

3. l'esame del rendiconto annuale preventivo e consuntivo (cann. $1284 \$ 3 ; 1287 \$ 1$ ): in proposito dovrebbe essere resa obbligatoria la presentazione anche del bilancio preventivo, come prevede il $\mathrm{CIC}$, in modo che si possa stabilire una progettualità della carità, a livello diocesano. Il Vescovo dovrà vigilare perché i preventivi della gestione economica Parrocchiale siano sempre analizzati dei Parroci e dei loro Consiglii per gli Affari Economici, come prevede il Codice (can. 537) per rendere possibile questa programmazione attraverso la condivisione delle risorse anche a livello delle comunità locali;

4. le licenze per eventuali atti di straordinaria amministrazione (ad esempio emanando un decreto ex can. $1281 \$ 2)^{16}$.

Con la funzione di vigilanza il Vescovo non si sostituisce agli amministratori, ma può invece offrire oltre ad un sostegno tecnicopastorale (ad esempio con occasioni di formazione per i membri dei Consigli per gli Affari Economici) anche un'azione di indirizzo all'uso caritativo dei beni ecclesiali.

Non si deve del resto dimenticare che, nei i casi gravi di negligenza, di uso improprio o personale dei beni e anche di altri problemi nella

\footnotetext{
${ }^{16}$ "Responsability for the administration of temporal goods, either at the diocesan level or within institutes of consecrated life has traditionally been considered to be a very important duty», così F.G. MORRISEY, Ordinary and extraordinary administration: can. 1277, The Jurist 48 (1989), pp. 709-726, a p. 709.
} 
gestione giuridico-pastorale dei beni, l'Ordinario potrà avocare a sé l'amministrazione (cann. $1279 \$ 1 ; 1740-1742 ; 1377$ e 1389).

La vigilanza in senso lato, deve dunque intendersi anche come una funzione fondamentale "di indirizzo" in materia di amministrazione dei beni, che il Vescovo eserciterà attraverso il Consiglio Diocesano per gli Affari economici (can. 492) e l’Economo diocesano (can. $494 \$ 1$ ): non possiamo perciò limitare questa funzione ad un mero controllo di carattere tecnico-amministrativo sui beni degli enti ecclesiastici sottoposti alla vigilanza del Vescovo ${ }^{17}$.

Per quanto detto è naturale dunque, attraverso un approfondimento della ratio di questa norma e dello spirito che sottintende tutte le altre disposizioni del Libro V del CIC, scoprire una caratteristica propositiva del compito di vigilanza del Vescovo diocesano: si tratta di attuare uno dei concetti del concetto che è a fondamento del Concilio Vaticano II, ossia la comunione che unisce i battezzati nel corpo ecclesiale e che quindi deve animare anche le relazioni tra enti canonici e l'amministrazione dei loro patrimoni/attività.

La rilevanza giuridica di questo tratto costitutivo della Chiesa venne ben evidenziata da Papa Giovanni Paolo II all'atto di promulgare il Codice di Diritto Canonico nel $1983^{18}$ :

"Lo strumento, che è il codice, corrisponde in pieno alla natura della chiesa, specialmente come viene proposta dal magistero del concilio Vaticano II in genere, e in particolar modo dalla sua dottrina ecclesiologica. Anzi, in un certo senso, questo nuovo codice potrebbe intendersi come un grande sforzo di tradurre in linguaggio canonistico questa stessa dottrina, cioè la ecclesiologia conciliare (omissis)... la dottrina per cui la chiesa è vista come "comunione", e che, quindi,

\footnotetext{
${ }^{17}$ Cfr. D. Le tourneau, Les Conseils pour les affaires économiques: origine, nature, Il Diritto Ecclesiastico 89 (1988), p. 613 ss. e M. Rivella, Partecipazione e corresponsabilità nella Chiesa, i consigli diocesani e parrocchiali, Milano 2000, p. 163.

${ }^{18} \mathrm{Si}$ possono approfondire le novità introdotte dal Concilio Vaticano II, e poi recepite dal Codice del 1983, nell'interessante analisi di R. Metz, Les responsables des biens des Ėglises dans la perspective de Vaticano II, comparée à celle du Code de 1917, Prawo Kanoniczne 20 (1977) n. 1-2, pp. 53-65.
} 
determina le relazioni che devono intercorrere fra le chiese particolari e quella universale, e fra la collegialità e il primato; la dottrina, inoltre, per la quale tutti i membri del popolo di Dio, nel modo proprio a ciascuno, sono partecipi del triplice ufficio di Cristo: sacerdotale, profetico e regale» ${ }^{19}$.

Questa precisazione dunque fa sì che nella funzione di vigilanza il Vescovo diocesano debba svolgere un ruolo di indirizzo e sollecitazione verso l'esercizio di opere di carità e assistenza ai più bisognosi del popolo a lui affidato: perché si realizzi attraverso l'attività delle diverse associazioni e aggregazioni ecclesiali ${ }^{20}$.

In questo ruolo di stimolo e orientamento il Vescovo però ha il compito fondamentale di assicurare che non si tratti di un semplice servizio sociale, magari in sostituzione delle carenze dell'attività statale. Ancora Papa Benedetto XVI nella lettera Apostolica "Sul servizio della Carità" scriveva:

«Pertanto, nell'attività caritativa, le tante organizzazioni cattoliche non devono limitarsi ad una mera raccolta o distribuzione di fondi, ma devono sempre avere una speciale attenzione per la persona che è nel bisogno e svolgere, altresì, una preziosa funzione pedagogica nella comunità cristiana, favorendo l'educazione alla condivisione, al rispetto e all'amore secondo la logica del Vangelo di Cristo. L'attività caritativa della Chiesa, infatti, a tutti i livelli, deve evitare il rischio di dissolversi nella comune organizzazione assistenziale, divenendone una semplice variante» ${ }^{21}$.

\footnotetext{
${ }^{19}$ Giovanni Paolo II, Sacrae disciplinae leges, AAS, 75 [1983] Pars II, XII.

${ }^{20}$ Diritto di associarsi riconosciuto dal Codice, ma anche dovere di attuare i fini della Chiesa, tra cui l'esercizio della carità. Cfr. su questo aspetto, F. G. MORRISEY, The right of association as a basic right of the faithful, in: W. Aymans(ed.), Das Konsoziative Element in der Kirche (St. Ottilien 1989), pp. 7-10.

${ }^{21}$ Benedetto XVI, Lettera Enciclica Deus caritas est, 25 dicembre 2005, AAS 98 (2006). In questo senso anche nel documento della Congregazione PER I VESCOvi, Direttorio pastorale dei vescovi Ecclesiae imago, 22-II-1973, al n. 133: «Il Vescovo procura con mezzi idonei che i fedeli vengano educati al senso della partecipazione e della collaborazione anche rispetto ai beni temporali, necessari alla Chiesa per conseguire il proprio fine: cosi tutti si sentiranno corresponsabili, ciascuno secondo
} 
Il Vescovo dovrà essere sempre attento alle reali necessità del popolo assicurando, col discernimento che è proprio del pastore, che le esigenze di carità e di assistenza dei più poveri non restino senza una risposta efficace da parte della Chiesa, rispettando dunque le caratteristiche e l'autonomia di governo delle diverse formazioni ecclesiali $^{22}$, potrà opportunamente orientarne l'agire e l'attività.

La vigilanza del Vescovo si esprime infine anche sulla regolamentazione dell'esercizio del culto pubblico, che direttamente o indirettamente tocca la sfera della gestione dei beni e delle risorse, manifestando e testimoniando un uso ecclesiale ed evangelico delle risorse economiche. Per esempio si dovrà vigilare, e se del caso evitare, iniziative liturgiche (per es. processioni) organizzate primariamente per raccogliere denaro non con scopi "chiaramente" caritativi o comunque evangelici; evitare, e se del caso vietare, processioni e iniziative liturgiche talvolta organizzate primariamente per raccogliere danaro. Arrivando anche a vietare la raccolta di danaro durante le processioni o altre manifestazioni a carattere religioso.

Stesso discorso vale per i regolamenti dei Santuari, luoghi dove non deve emergere il sospetto del commercio (cfr. can. 1232). Laddove diffuso, ad esempio, deve essere abbandonato l'uso di legare l'esercizio delle benedizioni di oggetti cui viene legata un'offerta che di fatto spesso è obbligatoria. Simile anche la tradizione in alcuni luoghi della benedizione delle tombe il giorno dei defunti, legata con offerta relativa, da stabilirsi comunque dall'Assemblea dei Vescovi della Provincia (can. 1264, 2).

Dunque si evidenzia la necessità di un esercizio in positivo della vigilanza da parte del Vescovo, che non potrà che rinsaldare sempre più il legame di comunione all'interno della Chiesa locale.

le sue capacità, del sostentamento economico della comunità ecclesiale e delle sue opere e beneficienze, nonché della conservazione, dell'incremento e della retta amministrazione dei beni della medesima».

${ }^{22}$ Can. $298 \$ 1$ e ss. 


\section{La funzione di incentivo alla carità attraverso gli organismi diocesani}

Anche attraverso gli organismi diocesani che presiede il Vescovo diocesano favorisce e sostiene iniziative ed opere di servizio al prossimo nella propria Chiesa particolare; egli può suscitare nei fedeli il fervore della carità operosa, che sia espressione di vita cristiana e di partecipazione alla missione della Chiesa, come anche troviamo nei due codici ai canoni 215 e 222 del CIC e canoni 25 e 18 del CCEO.

Come accennato questa funzione di incentivo verso la carità, il Vescovo la eserciterà in primo luogo con la presidenza del Consiglio Diocesano per gli Affari economici (can. 492) e degli altri organismi diocesani, come il Collegio dei Consultori. Potrà anche, se necessario, favorire la creazione di nuove strutture pastorali deputate a favorire l'azione caritativa, a livello di parrocchie e di altri degli enti che fanno capo alla diocesi (can. 1280).

Attraverso il Consiglio Presbiterale potrà poi svolgere una preziosa opera di esortazione e di sprone per i parroci e i sacerdoti della sua diocesi.

Per la pastorale dei Parroci in primo luogo dovrà ${ }^{23}$ :

- Orientare, regolamentare e vigilare sulla raccolta delle offerte in occasione di sacramenti: l'offerta dei fedeli è sempre dono in tutte le circostanze e va evitato il collegamento tra sacro e denaro (cfr. cann. 848, 1181 e 1264). Si ricorra dunque all'esortazione pastorale, onde educare alla collaborazione alla vita della Chiesa.

- Avviare iniziative perché le parrocchie organizzino gruppi di fedeli che sostengano stabilmente l'esercizio della carità verso i poveri, gli ultimi. Questo si può fare anche con opere di sensibilizzazione dei fedeli verso i bisogni dei Paesi più poveri, delle terre di missione, delle diocesi più povere, nonché seguendo le iniziative che la Santa Sede promuove per la riduzione del debito estero.

${ }^{23}$ V. DE PAOLIs, Il consiglio per gli affari economici ed i beni patrimoniali della Parrocchia, in: AA.VV., La Parrocchia, Città del Vaticano 1997, pp. 267-288. 
- I beni a disposizione delle Parrocchie non siano lasciati inutilizzati e l'utilizzo, orientato sempre dal Vescovo, primo amministratore della diocesi come detto, avvenga a preferenza a scopi solidali (associazioni di beneficienza, oltre alla Caritas parrocchiale, iniziative di sostegno a progetti di sviluppo nei paesi più poveri, ecc. $)^{24}$.

Il Vescovo dovrà poi esortare tutti i sacerdoti della sua diocesi ad una vita di stile "evangelico": attraverso lo stile personale dei presbiteri, si comunica ai fedeli il valore della scelta della carità. Come ha ricordato Papa Francesco, per esempio evitare il possesso di beni costosi (auto e altro) e di comodità esagerate, dando l'esempio di uno stile di vita sobrio.

Nell'ambito della sua azione di sostegno e orientamento dell'opera caritativa dei Parroci e dei sacerdoti, il Vescovo non potrà dimenticare un'attenzione particolare ai preti anziani, soli, ammalati, cercando di creare case di riposo (purtroppo ancora molto poche, spesso per i problemi economici connessi a sostenere tali iniziative) e offrendo altresì la possibilità ai sacerdoti di esperienze di vita comune.

\section{Conclusioni}

Dunque l'azione pastorale per la carità rimane una missione fondamentale del Vescovo che nell'Enciclica Deus Caritas Est Papa Benedetto XVI ha sollecitato con queste parole:

"Alla struttura episcopale della Chiesa, poi, corrisponde il fatto che, nelle Chiese particolari, $i$ Vescovi quali successori degli Apostoli portino la prima responsabilità della realizzazione, anche nel presente, del programma indicato negli Atti degli Apostoli (cfr 2, 42-44): la Chiesa in quanto famiglia di Dio deve essere, oggi come ieri, un luogo di aiuto vicendevole e al contempo un luogo di disponibilità a servire anche coloro che, fuori di essa, hanno bisogno di aiuto» ${ }^{25}$.

\footnotetext{
${ }^{24}$ J. E. LyNCH, The parochial ministery in the new code of canon law, The Jurist 42 (1983), pp. 383-421.

${ }^{25}$ Benedetto XVI, Lettera Apostolica in forma di Motu proprio, Sul servizio della Carità, 11 novembre 2012, Città del Vaticano, LEV.
} 
Questa mentalità riporta la centralità dell'esperienza della Chiesa Apostolica:

"Nessuno diceva sua proprietà quello che gli apparteneva, ma ogni cosa era fra loro comune. (...) Nessuno infatti tra loro era bisognoso, perché quanti possedevano campi o case li vendevano, portavano l'importo di ciò che era stato venduto e lo deponevano ai piedi degli apostoli; e poi veniva distribuito a ciascuno secondo il bisogno" (At 4,32.34-35) ${ }^{26}$.

\section{Bishop as witness of charity. Canonical profile}

The diocesan Bishop governs the particular Church entrusted to him with legislative, executive and judicial power (can. $391 \$ 1$ ), as well as through the exercise of his pastoral office, he, the Bishop is the first on which looms this charitable mission. It is a juridical requirement of the Bishop that the code of canon law and the documents of the Magisterium treat several times, from a pastoral point of view.

It is, in fact, inherent in the task of guiding the particular Church entrusted to him and guarantor of the ecclesial spirit, that the Bishop orients towards charity the action of priests, the faithful and associations of the Church (can. $318 \$ 1$ ), showing the attention to the poor and needy of the portion of the people of God that he presides.

This supporting role towards charity, the Bishop may exercise through the diocesan councils which he presides: the Diocesan Council for the Economic Affairs (can. 492), the Diocesan Pastoral Council and eventually other Diocesan organisms that could be constituted for this end, such as Caritas, organizations that must be created both, at the level of parishes, and other entities that are part of the head of the diocese (can. 1280).

The supervision of the management of the goods that competes to the diocesan Bishop in the light of the rules and of the Magisterium, should then be understood not only as a mere work of control, but also as a core function

26 "Le opere di carità derivano il loro senso dalla «carità-agape», cioè dall'amore che Dio ha avuto per noi in Gesù Cristo e che si è rivelato pienamente nel mistero della Pasqua", da V. De Paolis, I beni temporali della Chiesa, ed. a cura di A. Perlasca, Bologna 2011, p. 303. 
in the field of administration of the goods for realising that "attention to the poorest", which is part of the practice of the Church's mission.

Parole chiave: Vescovo; carità; economia; Papa; diocesi; beni temporali; gestione; vigilanza; parrocchie; beneficenza

KeY wORDS: Bishop; charity; economy; Pope; diocese; temporal goods; administration; supervisory; parishes

\section{Nota o Autorze:}

Prof. Antonio Interguglielmi - sacerdote della diocesi di Roma, professore di diritto canonico dello Studium Theologicum Galilaeae di Israele, affiliato alla Facoltà di Teologia della Pontificia Università Lateranense di Roma, ha pubblicato nel 2012 un libro nella Collana giuridica della LEV, $i$ Decreti singolari, dedicato all'attività amministrativa della Diocesi, e diversi articoli soprattutto di diritto canonico amministrativo e penale. E' Direttore dell'Ufficio per le Aggregazioni Laicali e le Confraternite della Diocesi di Roma e amministratore di diversi Enti ecclesiastici e Fondazioni della stessa Diocesi. 